\title{
EVALUACIÓN DEL APLANAMIENTO CONDILAR EN PACIENTES DENTADOS Y NO DENTADOS MEDIANTE RADIOGRAFÍA PANORÁMICA
}

\author{
EVALUATION OF CONDYLAR FLATTENING IN DENTATE AND \\ NON-DENTATE PATIENTS USING PANORAMIC RADIOGRAPHY
}

\author{
Anthony Cantor-Mora'* (1) \\ anthonycantor90@gmail.com
}

\section{Gustavo Adolfo Fiori-Chíncaro ${ }^{2}$} gfiori@ilaeperu.com

Artículo recibido: 10/02/2020

Arbitrado por pares

Artículo aceptado: 30/03/2020

* Autor corresponsal:

Anthony Cantor-Mora

anthonycantor90@gmail.com

\section{RESUMEN}

Introducción: Las alteraciones morfológicas de los cóndilos se determinan por la disminución de tejido óseo en el cóndilo mandibular, lo que cambia su forma y tamaño. Entre los signos más frecuentes encontramos el aplanamiento condilar, que se presenta entre los procesos dinámicos. Los cambios morfológicos más frecuentes son aplanamiento condilar, caracterizado por la pérdida de la convexidad del cóndilo articular. Radiográficamente, se presenta como una superficie convexa, la cortical ósea condilar es continua con ausencia de zonas radiopacas o radiolúcidas irregulares. Objetivos: Evaluar las características radiográficas de aplanamiento condilar de la articulación temporomandibular en pacientes dentados y no dentados mediante el uso de la radiografía panorámica. Métodos: Se evaluaron 520 radiografías panorámicas (260 de pacientes dentados y 260 no dentados), de pacientes de ambos sexos, entre 18 a 70 años, que fueron tomadas en el Centro de Radiología Digital Oral y Maxilofacial D3RX de Floridablanca (Colombia), entre enero de 2017 y diciembre de 2018, elegidos de forma aleatoria simple. La muestra fue definida mediante una base de datos de estudios científicos referenciados bibliográficamente, y mostró en este estudio una población evaluada con más alto promedio. Resultados: Los pacientes desdentados tienen mayor incidencia a presentar alguna alteración en la forma, el lado y el tipo de aplanamiento según la radiografía panorámica. Conclusiones: Los cambios morfológicos de los cóndilos observados en la radiografía panorámica según género mostró mayor prevalencia en mujeres que en hombres, mayor incidencia en cóndilos redondeados y afectación mayor en pacientes no dentados. Los resultados de este estudio muestran que, a pesar de que la radiografía panorámica no es considerada la mejor herramienta para determinar la presencia de cambios morfológicos leves a nivel de la mandíbula, puede ser utilizada por el odontólogo como la primera ayuda diagnóstica para obtener una visión general no solo de la articulación temporomandibular, sino de otras estructuras anatómicas y dentarias que deben ser estudiadas en trastornos de la articulación temporomandibular.

Palabras claves: cóndilo, articulación temporomandibular, panorámica

\begin{abstract}
Introduction: Morphological alterations of the condyles are determined by a decrease of bone tissue in the mandibular condyle, changing its shape and size. One of the most frequent signs of these alterations involves condylar flattening that occurs among dynamic processes and is characterized by a loss of convexity of the articular condyle. Radiographically this is observed as a convex surface, the condylar bone cortex is continuous and there is an absence of irregular radiopaque or radiolucent areas. Objectives: To evaluate the radiographic characteristics of condylar flattening of the temporomandibular joint in dentate and non-dentate patients using panoramic radiography. Methods: We evaluated panoramic radiographs obtained at the D3RX Oral and Maxillofacial Digital Radiology Center in Floridablanca-Colombia from January 2017 to December 2018.

Patients were randomly selected according to fulfillment of the following inclusion criteria:
\end{abstract}

Citar como: Cantor-Mora A, FioriChíncaro GA. Evaluación del aplanamiento condilar en pacientes dentados y no dentados mediante radiografía panorámica. Rev Cient Odontol (Lima). 2020; 8(1): e006.

DOI: 10.21142/2523-2754-0801-2020-006

\footnotetext{
1 Facultad de Odontología, Universidad Santo Tomás. Bogotá, Colombia.

2 Instituto Latinoamericano de Altos Estudios en Estomatología (ILAE). Lima, Perú.
} 
availability of panoramic radiographs showing the entire condylar structure of male and female patients aged 18 to 70 years attending from January 2017 to December 2018. This number of radiographs was defined according to a database. We evaluated the presence of flattening, the shape and the affected side and sex. The Chi-square test was applied. $\mathrm{P}<0.005$. Results: A total of 520 panoramic radiographs were obtained; 260 from dentate patients and 260 from non-dentate patients. Patient characteristics show that edentulous patients had a higher incidence of alterations in the shape, side and type of flattening in the panoramic radiographs. Edentulous patients have a higher incidence of presenting some alteration in their shape, side and flattened condyles observed in panoramic radiographs in $51.2 \%$ versus $35.1 \%$ of dentate patients $(\mathrm{P}<0.001)$. Conclusions: The morphological changes of the condyles observed in panoramic radiographs were more prevalent in women than men, with a higher incidence in rounded condyles and greater involvement in edentulous patients. The results of this study show that although panoramic radiography is not considered the best tool to determine the presence of slight morphological changes at the level of the jaw, it can be used as the first diagnostic aid to obtain an overview of not only the temporomandibular joint but also other anatomical and dental structures that should be studied in disorders of the temporomandibular joint.

Keywords: condyle, temporomandibular joint, panoramic

\section{INTRODUCCIÓN}

Las alteraciones morfológicas de los cóndilos se determinan por la disminución de tejido óseo en el cóndilo mandibular, lo que cambia su forma y tamaño. Entre los signos más frecuentes encontramos el aplanamiento condilar, que presenta cambios entre los procesos dinámicos y la capacidad de reparación de los tejidos orgánicos. Existen 3 tipos de remodelación: progresiva, regresiva y circunferencial $\left.{ }^{(2-9}\right)$.

La etología de los cambios morfológicos se ha relacionado con la artritis reumatoide, el trastorno interno del ATM, el uso de esteroides, los tratamientos ortodónticos y la cirugía ortognática. Esto puede causar estabilidad esquelética, oclusal, deformidad dentofacial y disfunción temporomandibular ${ }^{(8-18)}$. El cambio morfológico más frecuente es el aplanamiento condilar, que se caracteriza por la pérdida de la convexidad del cóndilo articular. Se considera aplanado un cóndilo que ha perdido el contorno redondeado de la superficie condilar. Una pérdida de convexidad en el cóndilo por preservación de la cortical ósea es considerado aplanamiento fisiológico; por el contrario, una pérdida de convexidad condilar con alteración o ausencia de la cortical ósea es un aplanamiento patológico. Un cóndilo normal se caracteriza por tener una proyección ósea redondeada con una biconvexidad superior y una superficie ovalada en el plano axial. Radiográficamente, se observa con una superficie convexa, la cortical ósea condilar es continua con ausencia de zonas radiopacas o radiolúcidas irregulares $\left.{ }^{5-24}\right)$.

La radiografía panorámica ha sido recomendada como una herramienta de diagnóstico en pacientes que presentan alteraciones de la ATM y puede ser utilizada para la determinación de los cambios óseos en el cóndilo o del proceso condilar. Es muy útil cuando el paciente no pueda abrir la boca a causa de trismos, traumatismo o no posee capacidad económica para acceder a un estudio de tomografía computarizada de haz cónico o una resonancia magnética. En esta radiografía se puede visualizar la rama de la mandíbula, la apófisis estiloides, el seno maxilar superior, la ATM y las estructuras de la boca que no pueden ser examinadas mediante una radiografía periapical. Entre sus ventajas está su costo accesible, el ser rutinario y ofrecer una imagen anatómica extensa con una dosis baja de radiación. Sin embargo, es importante considerar desventajas como la sobreproyección de las imágenes, la visión bidimensional de una estructura tridimensional y la poca capacidad de observar cambios menores $\left({ }^{32-40}\right)$. El propósito de este estudio fue evaluar las características radiográficas de aplanamiento condilar de la articulación temporomandibular en pacientes dentados y no dentados, mediante el uso de la radiografía panorámica. Esta detección permitiría el tratamiento ideal por parte de los especialistas, lo que genera cambios y mejoría en quienes presentan esta condición. 


\section{MATERIALES Y MÉTODOS}

Se realizó un estudio descriptivo, observacional, transversal y retrospectivo. Se evaluaron 520 radiografías panorámicas que fueron tomadas en el Centro de Radiología Digital Oral y Maxilofacial D3RX de Floridablanca (Colombia), entre enero de 2017 y diciembre de 2018.

Se eligieron 260 radiografías panorámicas en pacientes dentados y 260 en no dentados, los cuales fueron seleccionados de manera aleatoria simple. Se consideró como dentados a aquellos pacientes con presencia de incisivos, caninos, premolares y primer y segundo molar de todos los cuadrantes, y como no dentados a aquellos con ausencia dental mínima de dos dientes posteriores ubicados en el mismo lado y que, a su vez, sea antagonistas uno del otro. La cantidad de radiografías fue definida mediante una base de datos de estudios científicos referenciados bibliográficamente.

Fueron excluidos pacientes con fracturas, traumatismos, tumoraciones benignas o malignas en la articulación temporomandibular, con aparatología fija de ortodoncia, con ausencia de dientes anteriores, imágenes con artefactos, con movimientos o imágenes con distorsión por mal posicionamiento del paciente. $\mathrm{El}$ investigador fue capacitado y calibrado por un radiólogo oral y maxilofacial con 5 años de experiencia, y se realizó una prueba piloto interexaminador, la cual tuvo un valor kappa de 0,9 .

Su objetivo fue evaluar y medir el grado de aplanamiento condilar de la articulación temporomandibular en pacientes dentados y no dentados mediante el uso de la radiografía panorámica. Se utilizó un equipo panorámico J Morita Veraviewepocs 2D HD modelo X550, japonés, con kV 70 y 10 Ma. Para la medición de los valores, se empleó el software de captura propio (i-Dixel), desarrollado directamente por J Morita, en el que los drivers de captura son diseñados específicamente para el equipo Veraviewepocs 2D HD. Las radiografías panorámicas digitales seleccionadas se proyectaron en un monitor LED de 29 pulgadas (Marca LG, modelo 22MK400H), con resolución 1920 x 1080, y fueron analizadas por un solo examinador.
Se realizaron medidas estandarizadas mediante trazos en un plano referencial horizontal tomando como referencia los siguientes trazos: una línea vertical desde el inicio de la imagen panorámica que pasa cerca del conducto auditivo externo y una línea horizontal que pase tangente a la cima condílea. Los trazos que se mencionaron anteriormente se trazan en la parte derecha e izquierda de la imagen panorámica; luego, se traza una línea de intersección de los trazos donde se realizará la medición del ángulo mediante el software i-Dixel del equipo J morita Veraviewepocs 2D HD. El ángulo generado por i-Dixel se determinó mediante los siguientes grados de medida:

a. Leve. Pérdida de $20^{\circ}$, medida de acuerdo con la angulación desde el punto más alto de la fosa mandibular del temporal hasta eminencia del temporal.

b. Moderado. Pérdida de $20^{\circ}$ a $49^{\circ}$, medida según la angulación desde punto más alto de la fosa mandibular del temporal hasta eminencia del temporal.

c. Severo. Pérdida de más del $50^{\circ}$, medida de acuerdo con la angulación desde punto más alto de la fosa mandibular del temporal hasta eminencia del temporal.

\section{RESULTADOS}

La distribución de la población, según edad y sexo, correspondió a 281 mujeres y 239 hombres. Los hombres presentaron una media de edad de 31,24 años (DE: 9,451) y las mujeres, de 33,56 años (DE: 10.606).

En la tabla 2, se observa la asociación entre la condición dental, sean dentados y no dentados, y la presencia de aplanamiento del cóndilo, con un aumento en la condición de aplanamiento en los pacientes no dentados del $51,2 \%$ versus el $35,1 \%$ de los dentados $(\mathrm{P}<0,001)$.

En la tabla 3, se observa que existe una asociación entre la condición dental y la forma del cóndilo mandibular, siendo la forma redondeada la más frecuente tanto para pacientes dentados como no dentados. Asimismo, también se puede apreciar que hubo un gran porcentaje de cóndilos de forma triangular observados en el 35,3\% 
Tabla 1. Distribución del sexo y la edad de la muestra evaluada

\begin{tabular}{cccccc} 
SEXo & $\mathbf{n .}^{\circ}$ & Media & D. E. & Mínimo & Máximo \\
Masculino & 239 & 31,24 & 9,451 & 18 & 50 \\
\hline Femenino & 281 & 33,56 & 10,606 & 18 & 50 \\
\hline
\end{tabular}

Tabla 2. Asociación entre la condición dental y el aplanamiento del cóndilo

\begin{tabular}{ccccc|} 
Condición & Recuento & Presente & $\begin{array}{c}\text { Aplanamiento } \\
\text { Ausente }\end{array}$ & Total \\
\hline \multirow{2}{*}{ Dentado } & n. & 122 & 226 & 348 \\
\hline \multirow{2}{*}{ Desdentado } & $\%$ & $35,1 \%$ & $64,9 \%$ & $100,0 \%$ \\
& n. & 88 & 84 & 172 \\
\hline \multirow{2}{*}{ Total } & $\%$ & $51,2 \%$ & $48,8 \%$ & $100,0 \%$ \\
\hline & $\%$ & 210 & 310 & 520 \\
\hline & $\%$ & $40,4 \%$ & $59,6 \%$ & $100,0 \%$ \\
\hline
\end{tabular}

$\mathrm{P}<0,00$ I

Prueba de Chi cuadrado

de pacientes dentados versus el $22,1 \%$ de no dentados $(\mathrm{p}=0,014)$.

En la tabla 4, se observó que existe asociación entre la condición dental y la afectación según el grado de aplanamiento del cóndilo mandibular, siendo la forma moderada la más frecuente en pacientes no dentados respecto de los dentados: 20,9\% contra 11,5\% ( $\mathrm{p}<$ $0,001)$.

\section{DISCUSIÓN}

La radiografía panorámica es la ayuda diagnóstica principal para realizar un tratamiento inicial en odontología, ya que es fácil de realizar, tiene un costo accesible y ofrece una imagen de la anatomía extensa a dosis bajas de radiación. La importancia de realizar un diagnóstico prematuro de los cambios morfológicos condilares de pacientes que presentan alguna alteración temporomandibular queda demostrada en esta investigación, en la que se pudo establecer la relación entre los cambios óseos; sin embargo, no en todas las radiografías observadas se pudo correlacionar estas manifestaciones $(1,16,34)$.

Algunas investigaciones (1, 4, 10) aseguran que los cambios morfológicos pueden ser analizados desde un punto de vista fisiológico, pues los cambios condilares
Tabla 3. Asociación entre la condición dental y la forma del cóndilo

\begin{tabular}{|c|c|c|c|c|c|c|}
\hline \multirow{2}{*}{ Condición } & \multirow{2}{*}{ Recuento } & \multicolumn{5}{|c|}{ Forma } \\
\hline & & Ovalada & Redondeada & Triangular & Trapezoidal & Total \\
\hline \multirow{2}{*}{ Dentado } & n. ${ }^{\circ}$ & 14 & 177 & 123 & 34 & 348 \\
\hline & $\%$ & $4,0 \%$ & $50,9 \%$ & $35,3 \%$ & $9,8 \%$ & $100,0 \%$ \\
\hline \multirow{2}{*}{ Desdentado } & n. ${ }^{\circ}$ & 12 & 104 & 38 & 18 & 172 \\
\hline & $\%$ & $7,0 \%$ & $60,5 \%$ & $22,1 \%$ & $10,5 \%$ & $100,0 \%$ \\
\hline \multirow{2}{*}{ Total } & n. ${ }^{\circ}$ & 26 & 280 & 161 & 52 & 520 \\
\hline & $\%$ & $5,0 \%$ & $53,8 \%$ & $31,0 \%$ & $10,0 \%$ & $100,0 \%$ \\
\hline
\end{tabular}

$P=0,014$

Prueba de Chi cuadrado

Tabla 4. Asociación entre la condición dental y el tipo de afectación del cóndilo

\begin{tabular}{cccccc|}
\hline Condición & No presenta & \multicolumn{5}{c|}{ Tipo de afección } \\
& Leve & Moderada & Severo & Total \\
\hline \multirow{2}{*}{ Dentado } & 229 & 71 & 40 & 8 & 348 \\
\hline \multirow{2}{*}{ Desdentado } & $65,8 \%$ & $20,4 \%$ & $11,5 \%$ & $2,3 \%$ & $100,0 \%$ \\
\hline \multirow{2}{*}{ Total } & 83 & 43 & 36 & 10 & 172 \\
\hline & $48,3 \%$ & $25,0 \%$ & $20,9 \%$ & $5,8 \%$ & $100,0 \%$ \\
\hline & 312 & 114 & 76 & 18 & 520 \\
\hline & $60,0 \%$ & $21,9 \%$ & $14,6 \%$ & $3,5 \%$ & $100,0 \%$ \\
\hline
\end{tabular}

$\mathrm{P}<0,00$ ।

Prueba de Chi cuadrado

observados en radiografías panorámicas posiblemente están relacionados con la respuesta que presenta el hueso ante el estrógeno y la prolactina; esto debido a que se ha encontrado una mayor cantidad de receptores para estas hormonas en el disco articular.

Alexiou et al. ( $\left.{ }^{31}\right)$ evaluaron a 114 pacientes a través de radiografías panorámicas y tomografías computarizadas de haz cónico, y encontraron alteraciones del trastorno temporomandibular en una media de edad de 46,62 años (DE: 13,65$)$. Takayama $\left({ }^{37}\right)$, en un estudio de evaluación de 970 pacientes con una edad media de 42,3 años (DE: 14.7), muestra hallazgos con respecto al cambio óseo en los lados izquierdo y derecho del cóndilo. Cuando se presentaron cambios óseos anormales en el cóndilo, la deformidad fue la más frecuente en los lados izquierdo y derecho de ambos grupos. Hirata et al. $\left({ }^{10}\right)$ sugieren que la incidencia e intensidad de TMD son más altas en sujetos con mayor pérdida de dientes en las zonas de soporte, independientemente de su sexo. Kranjcic et al. $\left({ }^{16}\right)$ indican que la falta de dientes mandibulares 
posteriores puede acelerar el desarrollo de la enfermedad articular degenerativa. Por el contrario, Gupta et al. $\left({ }^{14}\right)$ sugieren que la pérdida del soporte oclusal asociada significativamente con la sensibilidad muscular y de la ATM se produjo solo en los hombres. Este estudio muestra una asociación entre la condición de dentados y no dentados con la presencia de aplanamiento del cóndilo, y encuentra aumentada la condición de aplanamiento en los pacientes no dentados en un $51,2 \%$ versus un $35,1 \%$ de los dentados $(\mathrm{P}<0,001)$.

Ladeira $\left({ }^{40}\right)$ estableció que el aplanamiento condilar fue el cambio morfológico más prevalente en las alteraciones generadas por la osteoartritis, con un $77,6 \%$ de prevalencia. Yasa $\left({ }^{37}\right)$ analizó la morfología del cóndilo mediante tomografías computarizadas de haz cónico, con respecto a la forma del cóndilo en cuatro paramentos (oval, triangular, redondeado y trapezoidal) de pacientes de clase I comparados con los de clase II, y halló diferencias $(\mathrm{p}>0,172)$. En el presente trabajo, se observó que existe una asociación entre la condición dental y la forma del cóndilo mandibular, con la forma redondeada como la más frecuente tanto para pacientes dentados como no dentados. Asimismo, se pudo apreciar un gran porcentaje de cóndilos de forma triangular en el $35,3 \%$ de pacientes dentados versus el $22,1 \%$ en el caso de los no dentados $(\mathrm{p}=0,014)$.

El aplanamiento condilar como trastorno temporomandibular es el resultado de varios factores como la oclusión, la posición anormal del cóndilo y el disco articular, los movimientos musculares excesivos y los factores fisiológicos, psicológicos y sistémicos. Se han realizado varios estudios epidemiológicos, anatómicos y de diagnóstico por imágenes sobre los factores relacionados con trastorno temporomandibular. La relación entre los síntomas de las alteraciones temporomandibulares y los criterios de edad, sexo, condición dental y oclusal han sido demostrados. Las relaciones oclusales se determinaron en función de la ubicación de los dientes restantes observados mediante radiografía panorámica $(5,9,17,30)$. Los resultados de este estudio se presentan en esa misma tendencia, pues se observó que existe asociación entre la condición dental y la afectación según el grado de aplanamiento del cóndilo mandibular, siendo la forma moderada la más frecuente para pacientes no dentados, pues se presenta en el 20,9\% contra el $11,5 \%$ en pacientes dentados $(\mathrm{p}<0,001)$.

El uso de métodos radiográficos como la tomografía computarizada y la resonancia magnética para investigaciones más completas ante alteraciones presentes en la articulación temporomandibular es considerado más específico y avanzado; sin embargo, en un estudio desarrollado para comparar tres métodos radiográficos, se concluyó que no hay diferencias en la precisión diagnóstica entre el examen panorámico, el lateral de cráneo y la tomografía sagital transversal de cráneo $\left.{ }^{1}\right)$.

\section{CONCLUSIONES}

Los cambios morfológicos de los cóndilos observados en la radiografía panorámica, según género, mostraron una mayor prevalencia en mujeres que en hombres, y una mayor incidencia en la presencia de cóndilos redondeados, seguidos de los de forma triangular, así como una mayor prevalencia de aplanamientos moderados en pacientes no dentados. Los resultados también muestran que, a pesar de que la radiografía panorámica no es considerada la mejor herramienta para determinar la presencia de cambios morfológicos leves a nivel de la mandíbula, puede ser utilizada por el odontólogo como la primera ayuda diagnóstica para obtener una visión general no solo de la articulación temporomandibular, sino de otras estructuras anatómicas y dentarias que deben ser estudiadas en trastornos de la articulación temporomandibular.

Contribución de autoría: Anthony Cantor-Mora ha participado en la elaboración del artículo, la recolección de la información, la redacción y la aprobación de la versión final, y Gustavo Fiori-Chíncaro ha participado como asesor y aprobación de la versión final.

Fuente de financiamiento: Autofinanciado.

Potenciales conflictos de interés: Los autores declaran no tener ningún tipo de conflicto de intereses. 


\section{REFERENCIAS BIBLIOGRÁFICAS}

1. Quevedo PM, Machado ME, Fasanella M. Evaluation of the mandibular morphology in patients with temporo mandibular dysfunction through panoramic radiography. Oral 2012; 41: 8458.

2. Demirturk Kocasarac H, Celenk P. Effectiveness of digital subtraction radiography in detecting artificially created osteophytes and erosions in the temporomandibular joint.Imaging Sci Dent. 2017; 47: 99-107. doi: 10.5624/isd.2017.47.2.99

3. Back K, Ahlqwist M, Hakeberg M, Dahlstrom L. Occurrence of signs of osteoarthritis/arthrosis in the temporomandibular joint on panoramic radiographs in Swedish women. Community Dent Oral Epidemiol. 2017; 45 (5): 478-84.

4. Abramowicz S, Simon LE, Susarla HK, Lee EY, Cheon $\mathrm{JE}$, Kim S, et al. Are panoramic radiographs predictive of temporomandibular joint synovitis in children with juvenile idiopathic arthritis? J Oral Maxillofac Surg. 2014; 72 (6): 1063-9.

5. Hintze H,Wiese M,Wenzel A. Comparison of three radiographic methods for detection of morphological temporomandibular joint changes: panoramic, scanographic and tomographic examination. Dentomaxillofac Radiol. 2009; 38 (3): 134-140.

6. Kurita H, Ohtsuka A, Kobayashi H, Kurashina K. Is the morphology of the articular eminence of the temporomandibular joint a predisposing factor for disc displacement? Dentomaxillofac Radiol. 2000; 29 (3): 159-62.

7. Hirsch C, Hoffmann J, Turp JC. Are temporomandibular disorder symptoms and diagnoses associated with pubertal development in adolescents? An epidemiological study. J Orofac Orthop. 2012; 73 (1): 6-8, 10-8.

8. Poveda-Roda R, Bagan J, Carbonell E, Margaix M. Diagnostic validity (sensitivity and specificity) of panoramic X-rays in osteoarthrosis of the temporomandibular joint. Cranio. 2015; 33 (3): 189-94. doi: 10.1179/2151090314Y.0000000018

9. Goulart DR, Muñoz P, Olate S, de Moraes M, Fariña R. No differences in morphological characteristics between hyperplastic condyle and class III condyle. Int J Oral Maxillofac Surg. 2015; 44 (10): 1281-6. doi: 10.1016/j.ijom.2015.05.018

10. Hirata FH, Guimaraes AS, Oliveira JX, Moreira CR, Ferreira ET, Cavalcanti MG. Evaluation of TMJ articular eminence morphology and disc patterns in patients with disc displacement in MRI. Braz Oral Res. 2007; 21 (3): 265-71.

11. Atkinson WB, Bates RE, Jr. The effects of the angle of the articular eminence on anterior disk displacement. J Prosthet Dent. 1983; 49 (4): 554-5.

12. Magnusson T, Karlsson C. Clinical impact of radiological examinations of patients with suspected temporomandibular disorders. Swed Dent J. 2002; 26 (2): 67-74.

13. Wakita T, Mogi M, Kurita K, Kuzushima M, Togari A. Increase in RANKL: OPG ratio in synovia of patients with temporomandibular joint disorder. J Dent Res. 2006; 85 (7): 62732. doi: $10.1177 / 154405910608500709$
14. Gupta D, Rashmi NC, Sheikh S, Pallagatti S, Goyal G, Singh R, et al. The prevalence, radiographic appearance, and characteristics of zygomatic air cell defects (ZACDs) in symptomatic temporomandibular joint disorder patients in North Indian population. Oral Maxillofac Surg. 2014; 18 (4): 453-7.

15. Lin H, Zhu P, Lin Y, Wan S, Shu X, Xu Y, et al. Mandibular asymmetry: a three-dimensional quantification of bilateral condyles. Head Face Med. 2013; 9: 42.

16. Kranjcic J, Vojvodic D, Zabarovic D, Vodanovic M, Komar D, Mehulic K. Differences in articular-eminence inclination between medieval and contemporary human populations. Arch Oral Biol. 2012; 57 (8): 1147-52.

17. Crow HC, Parks E, Campbell JH, Stucki DS, Daggy J. The utility of panoramic radiography in temporomandibular joint assessment. Dentomaxillofac Radiol. 2005; 34 (2): 91-5.

18. Alomar X, Medrano J, Cabratosa J, Clavero JA, Lorente M, Serra I, et al. Anatomy of the temporomandibular joint. Semin Ultrasound CT MR. 2007; 28 (3): 170-83.

19. Masood F Katz JO, Hardman PK, Glaros AG, Spencer P. Comparison of panoramic radiography and panoramic digital subtraction radiography in the detection of simulated osteophytic lesions of the mandibular condyle. Oral Surg Oral Med Oral Pathol Oral Radiol Endod. 2002; 93 (5): 626-31.

20. Reid KI Greene CS. Diagnosis and treatment of temporomandibular disorders: an ethical analysis of current practices. J Oral Rehabil. 2013; 40 (7): 546-61.

21. Schmitter M, Wacker K, Pritsch M, Giannakopoulos NN, Klose C, Faggion C, Kress B, Leckel M, Rammelsberg P. Preliminary longitudinal report on symptom outcomes in symptomatic and asymptomatic women with imaging evidence of temporomandibular joint arthritic changes. Int J Prosthodont. 2010; 23 (6): 544-51.

22. Dantas JFC, Nogueira Neto JN, Sarmento VA, Campos PSF. Temporomandibular joint reconstruction after condylar fracture complication related to osteosynthesis material. Int J Oral Maxillofac Surg. 2018; 47 (1): 137-9.

23. Durham J, Wassell RW. Recent advancements in temporomandibular disorders (TMDs). Rev Pain. 2011; 5 (1): 18-25. doi: 10.1177/204946371100500104

24. Winocur E, Reiter S, Krichmer M, Kaffe I. Classifying degenerative joint disease by the RDC/TMD and by panoramic imaging: a retrospective analysis. J Oral Rehabil. 2010; 37 (3): 171-7. doi: 10.1111/j.1365-2842.2009.02035.x

25. Kurita H, Uehara S, Yokochi M, Nakatsuka A, Kobayashi H, Kurashina K. A long-term follow-up study of radiographically evident degenerative changes in the temporomandibular joint with different conditions of disk displacement. Int J Oral Maxillofac Surg. 2006; 35 (1): 49-54.

26. Manfredini D, Guarda-Nardini L, Winocur E, Piccotti F, Ahlberg J, Lobbezoo F. Research diagnostic criteria for temporomandibular disorders: a systematic review of axis I epidemiologic findings. Oral Surg Oral Med Oral Pathol Oral Radiol Endod. 2011; 112 (4): 453-62. 
27. Epstein JB, Caldwell J, Black G. The utility of panoramic imaging of the temporomandibular joint in patients with temporomandibular disorders. Oral Surg Oral Med Oral Pathol Oral Radiol Endod. 2001; 92 (2): 236-9.

28. Roman M, Kaplan K, Block P.Temporomandibular joint internal derangement score (TIDS): novel magnetic resonance imaging assessment score and its relation to invasive treatment in patients with clinical temporomandibular joint pathology. Oral Surg Oral Med Oral Radiol Endod. 2018; 4 (11): e00916.

29. Cortes D, Marholz C,Millas R, Moncada G. Bone manifestations of degenerative diseases in temporomandibular joint with internal derangements. Tomography study. Act Odontol Venez. 2013; 51 (2).

30. Emshoff R, Brandlmaier I, Gerhard S, Strobl H, Bertram S, Rudisch A. Magnetic resonance imaging predictors of temporomandibular joint pain. J Am Dent Assoc. 2003; 134 (6): 705-14. doi: 10.14219/jada.archive.2003.0256

31. Alexiou K, Stamatakis H, Tsiklakis K. Evaluation of severity of temporomandibular joint osteoarthritic changes related to age using cone bean computed tomography. Dentomaxillofac Radiol. 2009; 38 (3): 141-7.

32. Wiese M, Svensson P, Bakke M, List T, Hintze H, Petersson A, Wenzel A. Association bewteen temporomandibular joint symptoms, signs, and clinical diagnosis using the RDC/ TMD and radiography findings in temporomandibular joint tomograms. J Orofac Pain. 2008; 38 (3): 239-51.
33. Aragão J, Silveira ML, Rocha C, dos Santos L, Prado F. Edentulousness in relation to remodeling of the gonial angles and incisures in dentate and edentate mandibles: morphometric study using the Image J sofware. Surg Radiol Anat. 2014; 36 (9): 889-94. doi: 10.1007/s00276-014-1335-1

34. Jung JK, Sohn WJ, Lee Y, Bae YC, Choi JK, Kim J.Morphological and cellular examinations of experimentally induced malocclusion in mice mandibular condyle. Cell Tissue Res. 2014; 355 (2): 35563. doi: 10.1007/s00441-013-1754-z

35. Ozkan A, Altug H, Senel B. Evaluation of articular eminence morphology and inclination in TMJ internal derangement patients with MRI. Int J Morphol. 2012: 740-4.

36. Calle E, Ballona P, Morales R. Relation of the of articular eminence inclination with morphological alterations of temporomandibular condylars. Kiru 2017; 14 (2): 127-34.

37. Takayama $\mathrm{Y}$, et al. Comparison of occlusal condition and prevalence of bone changes in the condyle of patients with and without temporomandibular disorders. Oral Surg Oral Med Oral Pathol Oral Radiol Endod. 2008; 105 (1): 104-12.

38. Sato H, Osterberg T, Ahlqwist M, Carlsson GE, Gröndahl HG, Rubinstein B. Association between radiographic findings in the mandibular condyle and temporomandibular dysfunction in an elderly population. Act Odontol Scand.1996: 54 (6): 384-90.

39. Silva MH, Fuentes FR. Morfometría del proceso condilar y rama de la mandíbula en individuos adultos de la ciudad de Temuco, Chile. Int J Morphol. 2004; 22 (2): 169-72.

40. Ladeira DB, da Cruz AD, de Almeida SM. Digital panoramic radiography for diagnosis of the temporomandibular joint: CBCT as the gold standard. Braz Oral Res. 2015; 29 (1): 1-7. 